\title{
Histogram-Enhanced Principal Component Analysis for Face Recognition
}

\author{
Ana-Maria Sevcenco and Wu-Sheng Lu \\ Dept. of Electrical and Computer Engineering \\ University of Victoria \\ sevcenco@engr.uvic.ca,wslu@ece.uvic.ca
}

\begin{abstract}
In this paper we present an enhanced principal component analysis (PCA) algorithm for improving the rate of face recognition. The proposed method modifies the image histogram to provide a Gaussian shaped tonal distribution in the face images, such that spatially the entire set of face images presents similar facial gray-level intensities while the face content in the frequency domain remains mostly unaltered. Computationally inexpensive, the algorithm proves to yield superior results when applied as a preprocessing step for face recognition. Experimental results are presented to demonstrate effectiveness of the proposed technique.
\end{abstract}

\section{Introduction}

Over the last decade, face recognition has been an active area of research in computer vision and one of the most successful applications of image analysis and understanding. Since the beginning of its expansion in early ' 90 s [1], considerable research endeavors have continued for enhancing the face recognition process. Starting from basic algorithms such as principal component analysis (PCA) [1], independent component analysis (ICA) [2], linear discriminant analysis (LDA) [3], and continuing with higher-complexity methods such as Laplacianfaces [4], recent approaches [5]-[9] strive to improve the recognition process by combining known basic techniques, including new pre-processing steps and modifying the existing steps.

The work of particular interest and relevant to the method proposed below is mainly found in [1] and [5] where PCA-based algorithms are involved in face recognition. Motivated by the recent results of Liao et al. [5], in which a filtering preprocessing step is applied to the conventional statistics-based methods such as PCA and ICA to improve the face recognition rate, in this paper we propose a histogram-based preprocessing step which can be utilized in a similar framework, at a very low computational cost. Experimental results are presented to demonstrate the effectiveness of the proposed technique.

\section{PCA and Whitening PCA: A Review}

PCA is among the most popular techniques used for image dimensionality reduction [1]. An impressive number of face recognition algorithms employs this eigendecomposition method. One of them is the whitenedface approach [5] which combines a whitening technique with PCA/ICA in order to increase the rate of face recognition.

\subsection{PCA}

The PCA [1] is an eigenface-based approach to face recognition that seeks to capture the variation in a collection of face images and uses this information to encode and compare images of individual faces. The eigenfaces are the eigenvectors of the covariance matrix of the set of face images, where each image is treated as a point in a high dimensional space.

Eigenfaces extract relevant facial information, which may or may not be directly related to human intuition of face features such as eyes, nose, and lips, by capturing statistical variation between face images. Therefore, eigenfaces may be considered as a set of features which characterize the global variation among face images. Other advantages of using eigenfaces are an efficient image representation using a small number of parameters and reduced computational and dimensional complexity.

Given a data set $\mathcal{D}$, also called training set, of $M$ face images of $K$ individuals, the eigenface-based algorithm proposed in [1] starts by transforming each $N \times N$ image in $\mathscr{D}$ into a vector $\Gamma_{i}$ of dimension $N^{2}$, by concatenating the image rows. The $K$ individuals are called members, each one having $L=M / K$ images in $\mathcal{D}$. Next, the average face $\Psi$ is computed as $\Psi=\frac{1}{M} \sum_{i=1}^{M} \Gamma_{i}$, and subtracted from each vector $\Gamma_{i}: \Phi_{i}=\Gamma_{i}-\Psi$. The data matrix is then formed as $A=\left[\begin{array}{lll}\Phi_{1} & \ldots & \Phi_{M}\end{array}\right]$ and the covariance matrix is constructed as 
$C=\frac{1}{M} \sum_{i=1}^{M} \Phi_{i} \Phi_{i}^{T}=\frac{1}{M} A A^{T}$. Instead of directly computing the eigenvectors $u_{i}$ and eigenvalues $\lambda_{i}$ of matrix $C$ of size of $N^{2}$ by $N^{2}$, which usually is an intractable task for typical image sizes, [1] proposed an indirect approach by finding the eigenvectors $v_{i}$ and eigenvalues $\lambda_{i}$ of a reduced-size matrix $L=A^{T} A$, and expressing the eigenvectors of matrix $C$ as

$$
u_{i}=\lambda_{i}^{-0.5} A v_{i} \text { for } i=1, \ldots, M
$$

The eigenvectors $u_{i}$ of covariance matrix $C$, called eigenfaces, are used to represent the face images from $\mathcal{D}$, so as to examine a new image as whether or not it is a face image and, if it is, whether it is a face of a member or a stranger (non-member).

Let $\tilde{U}$ be the matrix composed of $p$ most significant eigenvectors (i.e. eigenfaces) that are associated with $p$ largest eigenvalues of $C$, and let $\Gamma$ represent a new image to be examined. First, the distance $d_{0}$ between the new image and the face space is computed as

$$
d_{0}=\left\|\Phi-\Phi_{f}\right\|_{2}
$$

where $\Phi=\Gamma-\Psi, \Phi_{f}=\tilde{U} \Omega=\tilde{U} \tilde{U}^{T} \Phi$ is the orthogonal projection of image $\Phi$ onto face space $\tilde{U}$ and $\Omega=\tilde{U}^{T} \Phi$ is the pattern vector of $\Phi$. Comparing the distance $d_{0}$ with a chosen threshold $\delta_{0}$, the new image $\Gamma$ can be classified as a face or non-face image.

Furthermore, if $\Gamma$ turns out to be a face image, it can be classified as a member or non-member face. And if it is a member then the individual member is identified. These are achieved by evaluating $d_{k}=\left\|\Omega-\Omega_{k}\right\|$ for $k=1, \ldots, K$ and comparing

$$
d_{\min }=\min _{k} d_{k}
$$

with a prescribed threshold $\delta_{1}$, where the class vector $\Omega_{k}$ is calculated as $\Omega_{k}=\frac{1}{L} \sum_{i=1}^{L} \Omega_{k}^{(i)}, k=1, \ldots, K$, and $\Omega_{k}^{(i)}$ is the pattern vector of the $i^{\text {th }}$ image of the $k^{\text {th }}$ member.

\subsection{Whitening PCA (WPCA)}

As demonstrated by Liao et al. [5], a pre-processing step of whitening and low-pass filtering, that flattens the power spectrum of face images and controls the noise at high frequencies, can improve recognition rate.

The motivation behind whitening technique resides in spectral behavior of natural scenes and facial images: their power spectra roughly fall with the increasing spatial frequency according to a power law $1 / f^{\alpha}$. This unbalanced power spectra may result in potential problems when used in searching for structural information in the image space, as the information at low frequencies may swamp the equally useful information at high frequencies [5]. The solution in [5] employs a whitening filter to attenuate the low frequencies and boost the high frequencies so as to yield a roughly flat power spectrum across all spatial frequencies, and a low-pass filter to control the noise at the high frequencies. This filtering component is integrated as a preprocessing step into the conventional PCA/ICA algorithms, and the entire method is called whitenedfaces recognition.

In [5], a low-pass filter with frequency response

$$
L(f)=\exp \left(-\left(f / f_{c}\right)^{n}\right)
$$

is applied in order to avoid increasing the noise amplitude in image, where $f_{c}=0.6 f_{\text {max }}, n=5$ and $f=\sqrt{u^{2}+v^{2}}$ is the absolute spatial frequency. Subsequently, the whitening filter is applied for balancing the power spectrum. Its frequency response has the expression

$$
W(f)=f^{\alpha_{\omega} / 2}
$$

where the optimal value of whitening parameter $\alpha_{\omega}$ is found to be 2.5. From (4) and (5), the whitening preprocessing is achieved by applying the combined filter as

$$
W_{L}(f)=W(f) L(f) .
$$

\section{Histogram-enhanced PCA (HPCA)}

Motivated by the work in [5], we propose a simple, yet effective preprocessing algorithm. Instead of adjusting the frequency content of the face image, here the enhancement takes place in the spatial domain by modifying the image histogram. Specifically, our objective is to obtain a more homogeneous tonal distribution for all the face images in $\mathcal{D}$, by "equalizing" the lighting conditions and intensities across the entire image set. As a result, the data set becomes more uniform in terms of facial gray level and light intensity level.

The histogram of a digital image with gray levels in range $[0,255]$ is a discrete function $h\left(r_{k}\right)=n_{k}$, where $r_{k}$ is the $k$ th gray level and $n_{k}$ is the number of pixels in the image having gray level $r_{k}$. For discrete values, the probability of occurrence of gray level $r_{k}$ in an image is approximated by $p_{r}\left(r_{k}\right)=n_{k} / n$ with $k=0, \ldots, 255$ and $n$ denoting the total number of pixels in the image.

Histograms are the basis for numerous spatial domain processing techniques, being straightforward to calculate 


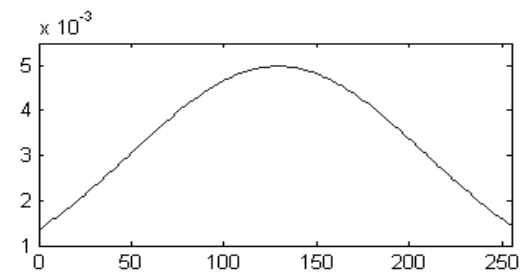

Figure 1. Gaussian shape of the imposed histogram.
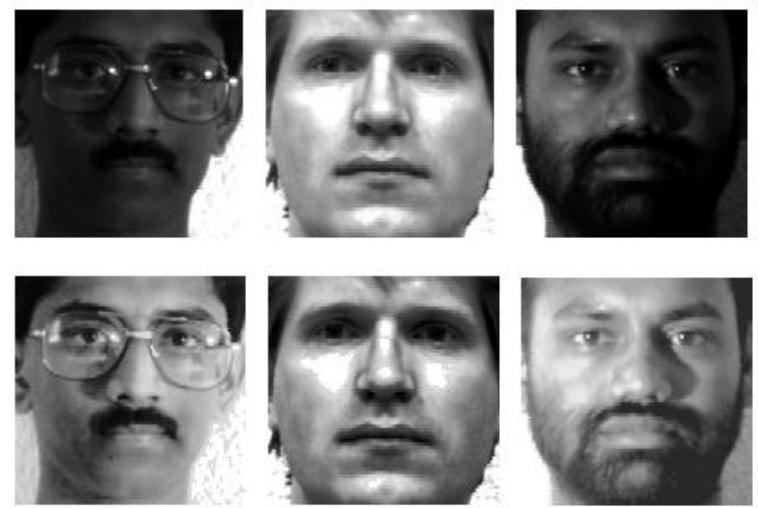

Figure 2. The effect of histogram-enhancing: original images (top row) and their processed counterparts (bottom row).

and having efficient hardware implementations for realtime image processing. Histogram-based techniques have been encountered in image processing applications such as image compression and segmentation.

For face recognition purposes, a helpful utility is the histogram cumulative distribution function (CDF) [10] which is defined by

$$
s_{k}=T\left(r_{k}\right)=\sum_{j=0}^{k} p_{r}\left(r_{j}\right)=\sum_{j=0}^{k} \frac{n_{j}}{n}, k=0, \ldots, 255 .
$$

Histogram matching [10] is the method used to generate a processed image that has a specified histogram. For a given image $I$ with histogram $h_{I}$, and a reference histogram $h_{R}$, the histogram matching can be achieved by (i) computing the CDFs of $h_{I}$ and $h_{R}$, denoted by $s_{I}$ and $s_{R}$, respectively; (ii) finding the correspondent gray levels $r_{R}$ from the reference histogram for which $s_{I}=s_{R}$; and (iii) assigning the values $r_{R}$ to $r_{I}$ in the original image histogram. In this way, the CDF of the original image, $s_{I}$, is matched to the reference CDF, $s_{R}$.

For improved face recognition, we propose to apply histogram matching for the tonal distribution in a face image to match a desired histogram $h_{R}$. For a natural and homogeneous appearance across the face images in data set $\mathcal{D}$, the Gaussian function
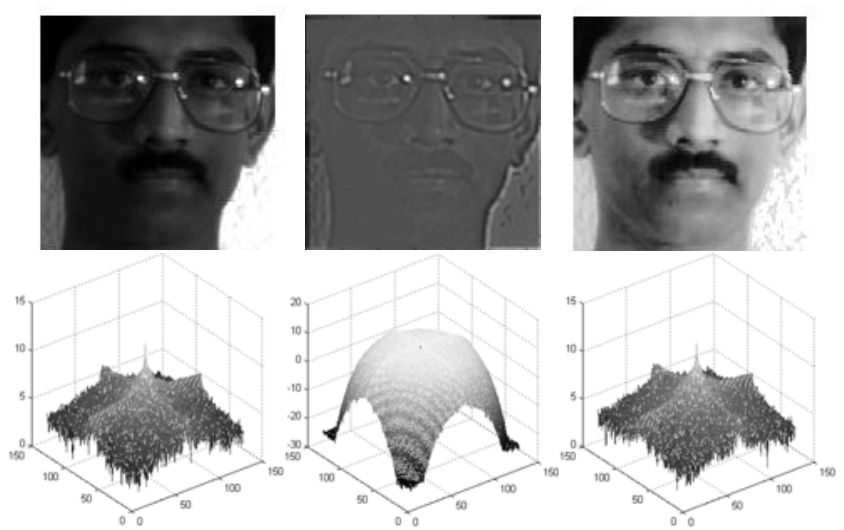

Figure 3. Top row from left to right: the original face image, its whitened version and its histogram-enhanced version.

Bottom row: their corresponding power spectra.
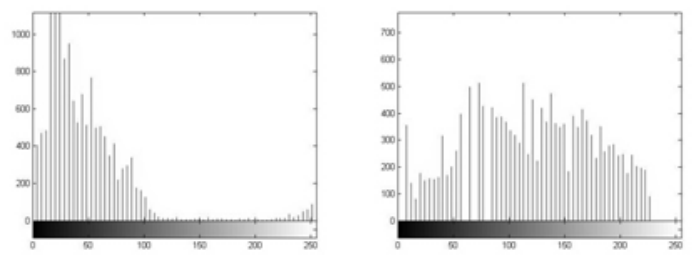

Figure 4. Histogram of original image (left) and its enhanced version (right).

$$
f(x)=a e^{-\frac{(x-b)^{2}}{2 c^{2}}}
$$

is chosen to be the desired reference histogram, where parameter $b$ is the position of the center of the peak, $c$ controls the width of the bell shape, and $a$ is the height of the curve's peak with $a=1 /(c \sqrt{2 \pi})$.

For example, a smooth tonal distribution of gray levels illustrated in Figure 1 can be obtained by choosing the center of the peak in the middle of the gray level scale to offer a balanced gray level distribution, i.e. $b=127$, width $c=80$ as it provides a wide range of middle gray level tones for a natural appearance of faces, and computing the corresponding height $a$. Applying the above histogram distribution to three different images in the first row of Figure 2 results in the second row in Figure 2. For comparison purpose, the effect in spatial and frequency domains of the whitening filter from (6) and the histogrambased processing using (8) is illustrated in Figure 3 using one of the test images. Using the same test image, Figure 4 shows how the distribution of the original dark image is changed approximating (but not perfectly matching) the shape of the imposed histogram as seen in Figure 1.

\section{A Case Study}


In the case study described below, the method proposed in Section 3 will be referred to as the histogram-based preprocessing PCA (HPCA). Our study aims to evaluate and compare the performance of HPCA with the existing WPCA and PCA algorithms under various conditions. For all the tests carried out, we chose to use the Yale face database [11] as it includes more images per class (subject) than other test data sets, such as FERET database. It contains a set of 165 grayscale images in GIF format of 15 subjects (Figure 5), with 11 poses per subject (Figure 6), namely center-light, with glasses, happy, left-light, without glasses, normal, right-light, sad, sleepy, surprised, and wink, denoted as pose 'a', 'b', ..., and 'k', respectively.

All the images have been cropped to a $128 \times 128$ pixel size, with the image center approximately placed between the 2 nostrils of subject's nose, as is illustrated in Figures 5 and 6 . The challenges arise not only in terms of variable lighting conditions and skin level intensities, which are addressed by utilizing the histogram-based preprocessing, but also in terms of different facial expressions, as can be observed in Figure 6.

Herein we carried out an examination more thorough than in [5] by investigating both face identification and face discrimination issues on several distinct training and testing sets, employing $p$ eigenvectors which usually were chosen as less than half or a quarter of the available $M$ eigenvectors. Implementation was done in MATLAB, employing the function 'histeq' for the histogram enhancing step, with the settings used to generate Figure 1.

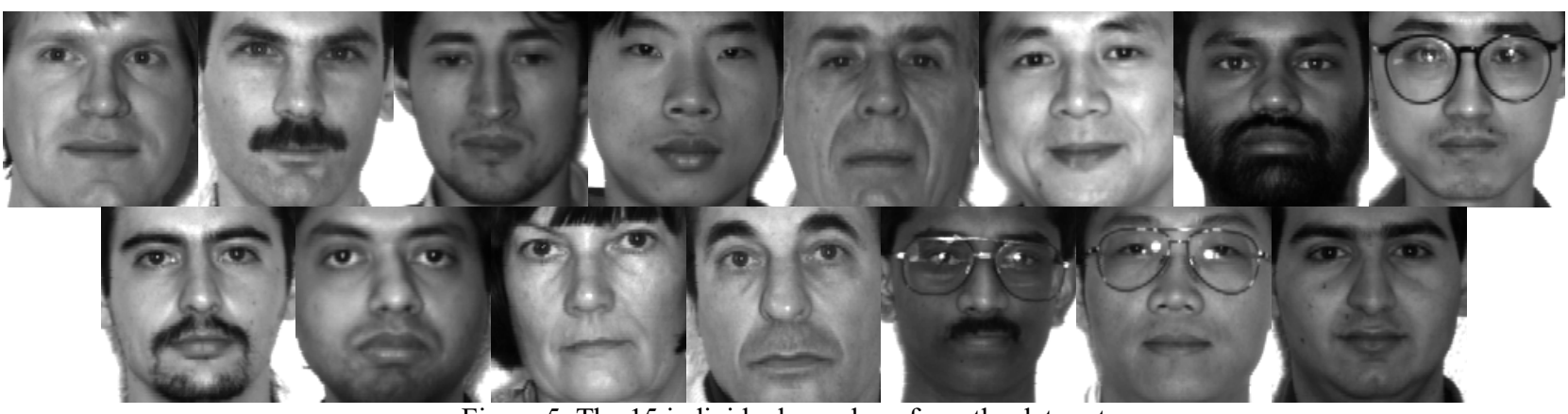

Figure 5. The 15 individual members from the data set

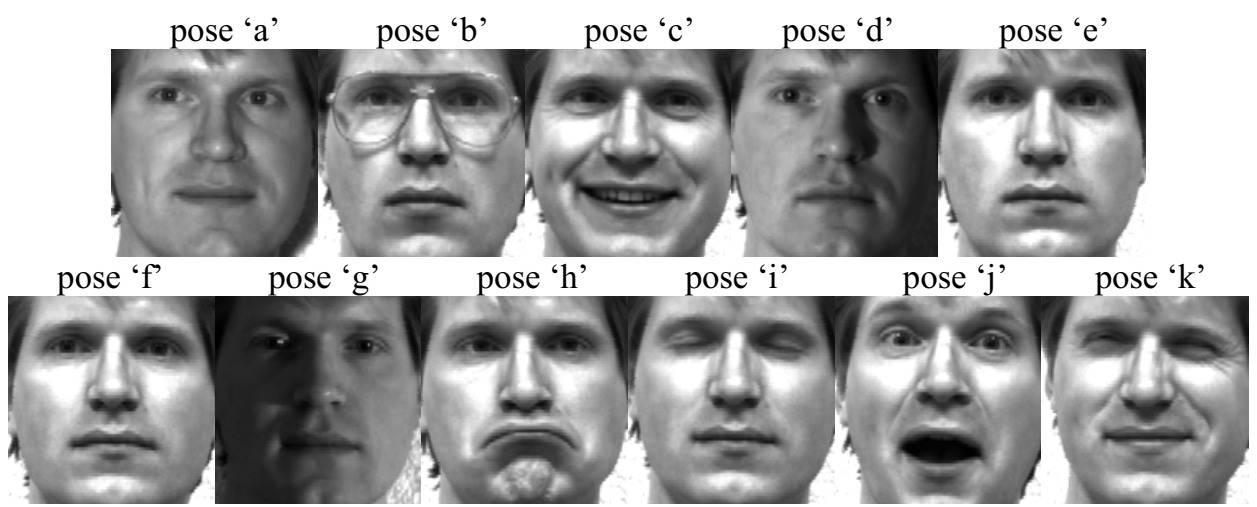

Figure 6 . The 11 poses of one member from the data set

Table 1. Numerical results for face/non-face and member/non-member discrimination

\begin{tabular}{|c|c|c|c|c|c|c|c|}
\hline \multirow{2}{*}{\multicolumn{2}{|c|}{ Image }} & \multicolumn{2}{|c|}{ PCA } & \multicolumn{2}{|c|}{ WPCA } & \multicolumn{2}{|c|}{ HPCA } \\
\hline & & $d_{0}$ & $d_{\min }$ & $d_{0}$ & $d_{\min }$ & $d_{0}$ & $d_{\min }$ \\
\hline \multirow{3}{*}{$\begin{array}{c}\text { non- } \\
\text { members }\end{array}$} & img8 & 5126.23 & 3314.66 & 89857.97 & 29902.46 & 6564.31 & 4122.79 \\
\hline & img11 & 6537.06 & 4924.31 & 73403.53 & 30969.52 & 6277.05 & 4039.31 \\
\hline & img15 & 4109.20 & 4600.91 & 74992.70 & 36138.53 & 4283.26 & 4209.01 \\
\hline non-face & airplane & 9997.15 & 5809.74 & 126505.40 & 24124.03 & 8001.44 & 5618.35 \\
\hline $\max \left(d_{0}^{(f)}\right)$ & $/ \max \left(d_{\min }^{(f)}\right)$ & 3406.10 & 5945.43 & 69905.64 & 51538.42 & 3658.66 & 3708.01 \\
\hline \multicolumn{2}{|c|}{ gap (\%) } & 17.11 & - & 4.77 & - & 14.59 & 8.20 \\
\hline
\end{tabular}

Table 2. Nine cases considered for face identification

\begin{tabular}{|c|c|c|}
\hline & Training Set: All 15 members with pose(s) & Testing Set: All 15 members with one pose \\
\hline Case 1 & 'a', 'c', 'd', 'e', 'g', 'h', 'i' & 'f' - normal pose \\
\hline
\end{tabular}




\begin{tabular}{|c|c|c|}
\hline Case 2 & 'a', 'd', 'e', 'g' & ' $\mathrm{f}$ ' - normal pose \\
\hline Case 3 & 'a', 'e' & ' $\mathrm{f}$ ' - normal pose \\
\hline Case 4 & 'a' & ' $\mathrm{f}$ ' - normal pose \\
\hline Case 5 & 'a', 'd', 'e', 'g' & 'c'- happy pose \\
\hline Case 6 & 'a', 'd', 'e', 'g' & 'h' - sad pose \\
\hline Case 7 & 'a', 'd', 'e', 'g' & 'i' - sleepy pose \\
\hline Case 8 & 'a', 'd', 'e', 'g' & 'j'-surprised pose \\
\hline Case 9 & 'a', 'd', 'e', 'g' & ' $\mathrm{k}$ ' - wink pose \\
\hline
\end{tabular}
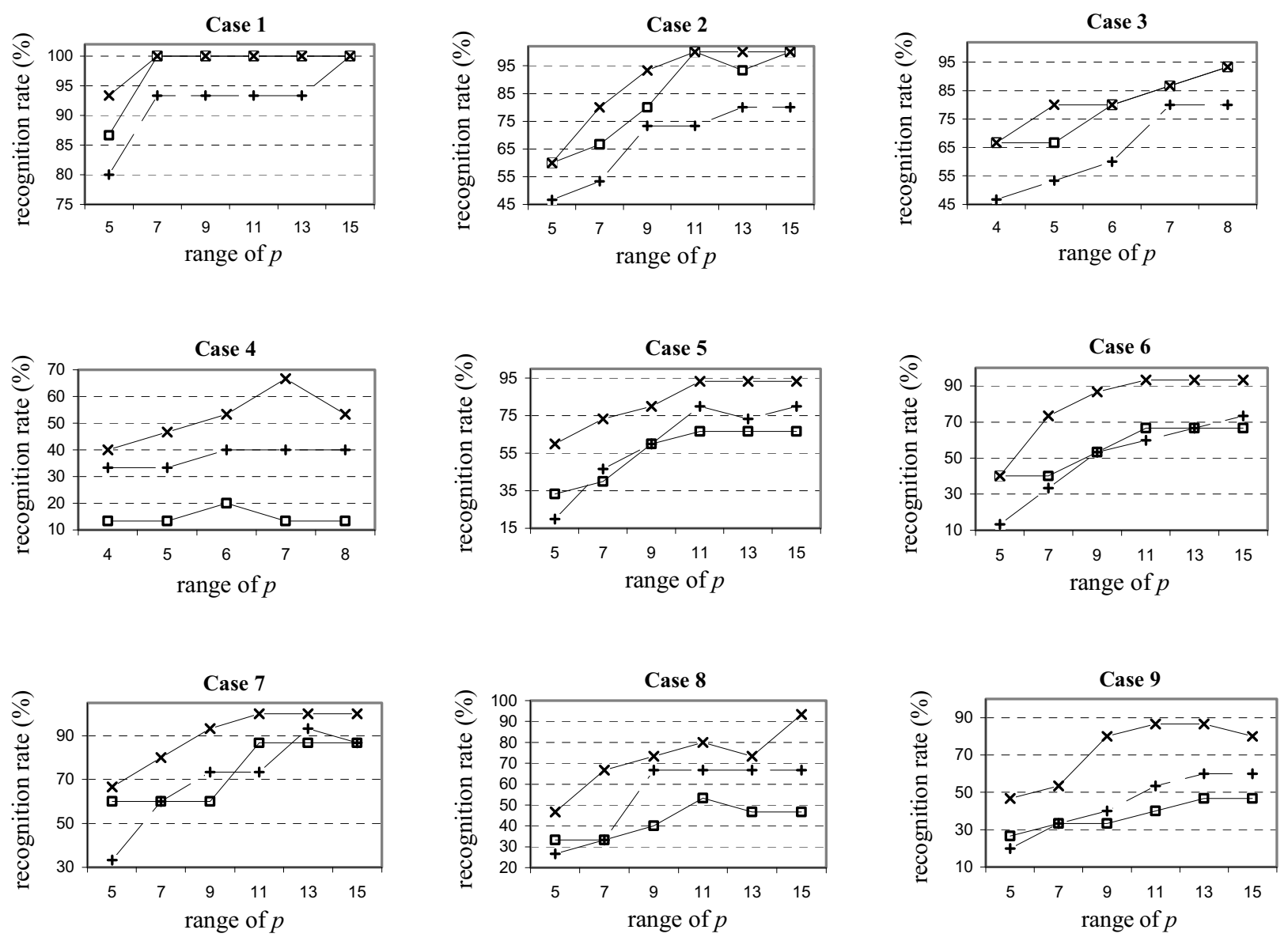

Figure 7. Comparison results for PCA (dashed line with plus), WPCA (solid line with square) and

HPCA (solid line with cross) for all nine cases

\subsection{Face/non-face and member/non-member discrimination}

The training set $\mathscr{D}$ used in this case had a total of $M=48$ images with $K=12$ members out of the 15 subjects, each one with $L=4$ poses: 'a', 'd', 'e' and 'g'. As a result, there were available $M=48$ eigenfaces from which a subset of $p=12$ was chosen to represent the face images.

A set of images, called testing set, was used to evaluate the discrimination performance of PCA, WPCA and HPCA. The test set consisted of pose ' $\mathrm{f}$ ' of all 15 individuals in the database, plus one non-face image called airplane. Among the 15 face images, there were 3 non-members, namely img8, img 11 and img 15 .

To evaluate the discrimination performance of the three methods, we introduce a measure called gap, which quantifies the distance between the class of non-face images $\boldsymbol{C}^{(n f)}$ and the class of face images $\boldsymbol{e}^{(f)}$ into the face space $\tilde{U}$. This measure is defined by

$$
\operatorname{gap}(\%)=\frac{\min \left(d_{0}^{(n f)}\right)-\max \left(d_{0}^{(f)}\right)}{\min \left(d_{0}^{(n f)}\right)} \cdot 100
$$


where $\min \left(d_{0}^{(n f)}\right)$ is the smallest $d_{0}$ defined by (2) for $\boldsymbol{e}^{(n f)}$ in $\mathcal{D}$, and $\max \left(d_{0}^{(f)}\right)$ denotes the largest $d_{0}$ for $\boldsymbol{e}^{(f)}$ in $\mathcal{D}$.

The above measure also applies for quantifying the distance between the class of non-member images $\boldsymbol{C}^{(\mathrm{nm})}$ and the class of member images $\boldsymbol{e}^{(m)}$ into the face space $\tilde{U}$, where the measure gap is defined in a similar way as

$$
\operatorname{gap}(\%)=\frac{\min \left(d_{\min }^{(n m)}\right)-\max \left(d_{\min }^{(m)}\right)}{\min \left(d_{\min }^{(n m)}\right)} \cdot 100
$$

where $\min \left(d_{\min }^{(n m)}\right)$ is the smallest $d_{\min }$ defined by (3) for $\boldsymbol{e}^{(n m)}$ in $\mathcal{D}$, and $\max \left(d_{\text {min }}^{(m)}\right)$ denotes the largest $d_{\text {min }}$ for $\boldsymbol{e}^{(m)}$ in $\mathcal{D}$.

Obviously, a bigger positive gap indicates easier discrimination between $\boldsymbol{e}^{(n f)}$ and $\boldsymbol{e}^{(f)}$, respectively $\boldsymbol{e}^{(n m)}$ and $\boldsymbol{e}^{(m)}$, while a negative value of this measure indicates that no discrimination can be done, as there is no gap but an overlapping. The evaluation results are summarized in Table 1 from which one can see that PCA offered the highest gap for face/non-face discrimination, but failed for members/non-members discrimination, WPCA provided only a small gap for face/non-face discrimination, while HPCA offered a reasonable gap for member/non-member discrimination and a substantial gap for face/non-face discrimination.

\subsection{Face identification}

For face identification, we investigated nine cases in which different poses of members were considered in forming the training and testing sets (Table 2).

Figure 7 illustrates the comparison results of the three methods in terms of recognition rate versus number of eigenfaces employed $(p)$, for the above nine cases. The plots in Figure 7 show how the recognition rate was improved by utilizing the HPCA algorithm when more than 3 eigenvectors were employed for image representation. We also note that the performance of HPCA algorithm was quite robust versus the number of images used in the training set. Indeed, even for a reduced training set, such as in Cases 3 and 4, the HPCA method outperformed the WPCA and PCA algorithms. A major improvement was achieved by HPCA when more difficult images were to be recognized, such as in Cases 5-9, while the WPCA algorithm yielded more false detections than the original PCA method.

In terms of computational complexity, on an average basis, the histogram-based image preprocessing was 1.3 times faster than the whitening image preprocessing, as it requires only a pixel intensity distribution as opposed to the two stages of filtering described in Section 2.2, equations (4), (5) and (6).

\section{Conclusion}

The histogram-enhancing method proposed in this paper can be used in combination with statistic-based methods and is shown to be a useful preprocessing tool for improving the face recognition rate, as demonstrated by the experimental results.

As future work we plan to develop a strategy of tuning the parameters of the desired Gaussian histogram for optimal face recognition performance.

\section{Acknowledgements}

The authors are grateful to NSERC for supporting this work and to Mr. Sergiu Sevcenco for his technical support.

\section{References}

[1] M.A. Turk, and A.P. Pentland, "Face recognition using eigenfaces", in Proc. IEEE Computer Society Conf. on Computer Vision and Pattern Recognition, 1991, pp. 586-591.

[2] M.S. Bartlett, J.R. Movellan, an T.J. Sejnowski, "Face recognition by independent component analysis", IEEE Trans. on Neural Networks, 2002, vol. 13, pp. 1450-1464.

[3] K. Etemad, and R. Chellappa, "Face recognition using discriminant eigenvectors", in Proc. IEEE ICASSP, 1996, vol. 4, pp. 2148-2151.

[4] X. He, S. Yan, Y. Hu, P. Niyogi, and H.-J. Zhang, "Face recognition using Laplacianfaces", IEEE Trans. on Pattern Analysis and Machine Intelligence, 2005, pp. 328-340.

[5] L.-Z. Liao, S.-W. Luo, and M. Tian, "'Whitenedfaces' recognition with PCA and ICA", IEEE Signal Processing Letters, 2007, vol. 14, pp. 1008-1011.

[6] F. Chichizola, L. De Giusti, A. De Giusti, and M. Naiouf, "Face recognition: reduced image eigenfaces method", ELMAR 47th International Symposium, 2005, pp. 159-162.

[7] K.J. Karande, and S.N. Talbar, "Simplified and modified approach for face recognition using PCA", International Conference on ICTES, 2007, pp. 523-526.

[8] K.-C. Kwak, and W. Pedrycz, "Face Recognition Using an Enhanced Independent Component Analysis Approach", IEEE Trans. on Neural Networks, 2007, vol. 18, pp. 530-541.

[9] X. Qing, and X. Wang, "Face Recognition using Laplacian+OPRA-faces", 6th World Congress on Intelligent Control and Automation, 2006, vol. 2, pp. 10013 -10016.

[10] R. C. Gonzalez, and R. E. Woods, Digital Image Processing, Second Edition, Prentice-Hall, New Jersey, 2002.

[11] Yale Face Database, Yale University, CT, USA. 